\title{
The first fruits of an HTP membrane platform: crystal structure of the CorA $\mathrm{Mg}^{2+}$ transporter
}

Vladimir V Lunin ${ }^{\dagger 1}$, Elena Dobrovetsky ${ }^{\dagger 2}$, Galina Khutoreskaya ${ }^{2}$, Rongguang Zhang 3 , Andrzej Joachimiak ${ }^{3}$, Declan A Doyle ${ }^{4}$, Alexey Bochkarev2,5,6, Michael E Maguire ${ }^{7}$, Aled M Edwards ${ }^{1,2,3,5,6}$ and Christopher M Koth*2,8

\begin{abstract}
Address: ${ }^{1}$ Department of Medical Biophysics, University of Toronto, 112 College Street, Toronto, ON, Canada, ${ }^{2}$ Banting and Best Department of Medical Research, University of Toronto, 112 College Street, Toronto, ON, Canada, ${ }^{3}$ Structural Biology Center \& Midwest Center for Structural Genomics, Biosciences Division, Argonne National Laboratory, 9700 S. Cass Av. Argonne, IL 60439, USA, ${ }^{4}$ Structural Genomics Consortium Botnar Research Centre, Oxford, Oxon OX3 7LD, UK, ${ }^{5}$ Department of Medical Genetics and Microbiology, University of Toronto, 112 College Street, Toronto, ON, Canada, ${ }^{6}$ Structural Genomics Consortium, Banting Institute, 100 College Street, Toronto, ON, Canada, ${ }^{7}$ Department of Pharmacology, Case Western Reserve University, Cleveland, OH 44106-4965, USA and ${ }^{8}$ Vertex Pharmaceuticals Incorporated, 130 Waverly St., Cambridge, MA 02139 USA

* Corresponding author †Equal contributors
\end{abstract}

from The 4th Recombinant Protein Production Meeting: a comparative view on host physiology

Barcelona, Spain. 2I-23 September 2006

Published: 10 October 2006

Microbial Cell Factories 2006, 5(SuppI I):SI9 doi:10.II86/1475-2859-5-SI-SI9

(c) 2006 Lunin et al; licensee BioMed Central Ltd.

Membrane proteins constitute $30 \%$ of prokaryotic and eukaryotic genomes but comprise a small fraction of the entries in protein structural databases. A number of features of membrane proteins render them challenging targets for the structural biologist, among which the most important is the difficulty in obtaining sufficient quantities of purified protein. We have developed robust procedures to express and purify large numbers of prokaryotic membrane proteins. Using a set of standard conditions, expression can be detected in the membrane fraction for approximately $30 \%$ of cloned targets. To date, over 30 membrane proteins have been purified in quantities sufficient for structural studies, typically in just two chromatographic steps. Theses include several transporters/ channels, sensor kinases, and rhomboid intramembrane proteases. Using this system, we have recently crystallized and solved the structure of the CorA magnesium transporter, the primary $\mathrm{Mg}^{2+}$ uptake system of most prokaryotes. Crystal structures of the full-length Thermotoga maritima CorA in an apparent closed state and its isolated cytoplasmic domain were determined at $3.9 \AA$ and $1.85 \AA$ resolution respectively. Our HTP strategy for membrane proteins, and the first structure from this effort, will be discussed. 